\title{
The Economic Implications of Burstiness of Cell Arrival Processes in Network Design
}

\author{
${ }^{1}$ Hilary Ugo Ezea, ${ }^{2}$ Candidus Okwor \\ ${ }^{1}$ Electrical/Electronic Engineering Department, ${ }^{2}$ Computer Engineering Department \\ ${ }^{1 \& 2}$ Federal University, Oye-Ekiti, Ekiti State, Nigeria
}

\begin{abstract}
Burstiness has a great influence on network architectures as well as network dimensioning. Hence, the knowledge of the different traffic models goes a long way in helping the network designer to make good choices concerning end buffer parameters, protocol selection as well as functional specifications, and QoS parameters. This paper takes a comparison of three traffic models- the Interrupted Poisson Process, the Interrupted Bernuolli Process and the ON-OFF traffic model with constant distribution. The comparison is based on buffer occupancy, cell delay and utilization; with the aim of investigating the economic implications of using these traffic models. The results of the simulation show that the ON-OFF (Constant distribution) and the $I P P$ have similar impacts which are far better than that of the IBP.
\end{abstract}

Index Terms: Burstiness, Traffic Model, IPP, IBP, ON-OFF

\section{Introduction}

With advancement in technology, networks are expected to support highly heterogeneous traffic sources. Therefore, it becomes imperative to take into consideration, the burstiness of traffic while designing networks. Burstiness can either be defined from the deterministic point of view or from the probabilistic point of view. However, most deterministic definitions of burstiness inherently lack statistical gains $[1,2,3]$. This implies that the network resources would be underutilized. On the other hand, probabilistic definition of burstiness, though it does not lack statistical gains, lacks the capability to effectively deal with the issue of longrange dependency [4]. In both, traffic characterization is the essential component. A traffic model is said to exist if there is a procedure for efficient and accurate inference for the parameters of the same mathematical structure [5]. Therefore it can be said that the efficiency in traffic characterization is given by the accuracy of the traffic model. The traffic characterization is the main information source for the right interpretation of network traffic [6]. A good traffic characterization should have among other properties, the following [7]:

- If two traffic flows are characterized according to say, C, then the aggregate of the flows should also be easily characterized according to $\mathrm{C}$.

- If a traffic characterized according to $\mathrm{C}$ is fed into a network element such as the entry node, the output flow should also be easily characterized according to $\mathrm{C}$ and both the queue size and the virtual delay should be easily characterized in the same framework as $\mathrm{C}$.

- The characterization according to $\mathrm{C}$ should be measureable and should also be able to capture the behavior of traffic in real networks as closely as possible without compromising the tractability

In broadband applications, burstiness is of great concern. This is because burstiness as well as correlation are two parameters that grossly affect QoS measures such as cell loss probability [8]. For instance, in Broadband Integrated Services Network B-ISDN, the ATM forum standardized the following parameters: peak cell rate, average cell rate or sustainable cell rate, cell delay variation for the peak rate, and maximum burst length. One can effectively police the peak rate using the peak rate and cell delay variation. Similarly, using the maximum burst length, one can estimate a cell delay variation that can be used to police the average rate. But these parameters are fairly inadequate when it comes to bandwidth allocation, since it can easily be shown that there are different distributions (arrival patterns or traffic models) with the same peak rate, average rate, and maximum burst length, but with different burstiness and inter-arrival correlations [8].

Burstiness includes these two factors: fluctuation in cell inter-arrival times and correlation in cell interarrival times [9]. Characterizing the burstiness of the cell arrival process using $C_{p}(z)$, which is a mixed index for the fluctuations in cell inter-arrival times as well as correlation of the cell inter-arrival times, was proposed in [10] as:
$\mathrm{C}_{\mathrm{p}}(\mathrm{z})=\sum_{\mathrm{k}=0}^{\infty} \frac{\mathrm{C}_{\mathrm{k}} \mathrm{z}^{\mathrm{k}}}{\left(\lambda^{-1}\right)^{2}}, \quad|\mathrm{z}|<1$
Eq. 1

Where: $\lambda^{-1}=$ the mean inter-arrival time, $\mathrm{C}_{\mathrm{k}}=$ correlation with lag $\mathrm{k}$ for the inter-arrival times.

$\mathrm{C}_{\mathrm{k}}=\mathrm{E}\left[\left(\mathrm{t}_{0}-\lambda^{-1}\right)\left(\mathrm{t}_{\mathrm{k}}-\lambda^{-1}\right)\right]$

Eq. 2

$\mathrm{t}_{0}, \mathrm{t}_{1}, \ldots$...being a sequence of inter-arrival times 
In the above equation, $\mathrm{C}_{0} /\left(\lambda^{-1}\right)^{2}$ expresses fluctuations in cell interarrival times and $\sum_{\mathrm{k}=1}^{\infty} \frac{\mathrm{C}_{\mathrm{k}} \mathrm{z}^{\mathrm{k}}}{\left(\lambda^{-1}\right)^{2}}$ expresses

correlations between cell inter-arrival times.

$\mathrm{C}_{\mathrm{p}}(\mathrm{z})$ includes the complete information for the second moment of the inter-arrival times. $\mathrm{C}_{\mathrm{p}}(0)$ for instance, provides a squared coefficient of variation and $\mathrm{dC}_{\mathrm{p}}(\mathrm{z}) / \mathrm{dz} \mathrm{I}_{\mathrm{z}=0}$ provides correlation of the consecutive inter-arrival times divided by the mean inter-arrival times. In particular, for a Poisson arrival process, $\mathrm{C}_{\mathrm{p}}(\mathrm{z})=1$, for all z. While for a renewal arrival process, $\mathrm{C}_{\mathrm{p}}(\mathrm{z})=$ (the squared coefficient of variation in the renewal process), for all z. For an MMPP, the $\mathrm{C}_{\mathrm{p}}(\mathrm{z})$ is derived in [9] and given as follows:

$\mathrm{C}_{\mathrm{p}}(\mathrm{z})=\lambda^{2}\left\{(1-\mathrm{z}) \theta \mathrm{R}^{-2}(\mathrm{z}) \mathrm{I}+\theta \mathrm{R}^{-2}(0)-\frac{\left(\theta \mathrm{R}^{-1}(0) \mathrm{I}\right)^{2}}{1-\mathrm{z}}\right\} \quad$ Eq. 3

Where:

$R(z)=(z-1) \Lambda+Q, I=(1, \ldots, 1)^{T}$, and $\theta=\left(\theta_{i}\right)$, denotes the probability vector, where $\theta_{i}$ is the probability that the state of the Markov chain $\theta$ is in phase $i$ at an arrival epoch.

Now considering the squared coefficient of variation in the different arrival processes which, as indicated above, is a component of the burstiness characterization of the cell arrival process. The coefficient of variation of the inter-arrival times by definition indicates the degree of randomness of the inter-arrival times. For instance, the coefficient of variation $(\mathrm{CoV})$ is the ratio of the standard deviation (square root of variance) to the mean value.

$\mathrm{CoV}=\frac{\text { Standard Variation }}{\text { Mean Value }}=\frac{\sqrt{\text { Variance }}}{\text { Mean Value }} \quad$ Eq. 4

Hence for a Poisson arrival process, the inter-arrival time is exponentially distributed with mean $\lambda^{-1}$ and standard deviation $\lambda^{-1}$. Consequently, the coefficient of variation is 1 . For a two-state MMPP, the interarrival times have a second-order hyper-exponential distribution which has coefficient of variation greater than 1. In [11], a 3- state Markovian arrival process MAP(3) is characterized as having coxian distribution of the inter-arrival times and as such, the coefficient of variation is less than 1. Similarly, for a Bernoulli arrival process, the inter-arrival time assumes a geometrical distribution (as already mentioned). But this geometric distribution has a mean $\frac{\mathrm{q}}{\mathrm{p}}$ and variance $\frac{\mathrm{q}}{\mathrm{p}^{2}}$. Therefore, the coefficient of variation of a Bernoulli arrival process is $\frac{1}{\sqrt{q}}$. Some basic distribution functions and their coefficient of variations are shown in table 1 .

All these variations suggest that the different traffic models and cell arrival processes have different effects on the burstiness parameter and consequently on the Quality of services.

Table 1: Some basic distribution functions and their coefficient of variations.

\begin{tabular}{|l|c|c|c|}
\hline $\begin{array}{l}\text { DISTRIBUTION } \\
\text { FUNCTION }\end{array}$ & MEAN & VARIANCE & CoV \\
\hline Poisson & $\lambda$ & $\lambda$ & $\frac{1}{\sqrt{\lambda}}$ \\
\hline Exponential & $\frac{1}{\lambda}$ & $\frac{1}{\lambda^{2}}$ & 1 \\
\hline Binomial & $\mathrm{Np}$ & $\mathrm{Npq}$ & $\frac{\sqrt{\mathrm{q}}}{\sqrt{\mathrm{Np}}}$ \\
\hline Geometric & $\frac{\mathrm{q}}{\mathrm{p}}$ & $\frac{\mathrm{q}}{\mathrm{p}^{2}}$ & $\frac{1}{\sqrt{\mathrm{q}}}$ \\
\hline
\end{tabular}

II. Traffic Model

Service and traffic models are very crucial for the widespread deployment of QoS oriented network services. This is because the tractability of network performance analyses is greatly determined by these service and traffic models [7]. Traffic models provide an insight into the behavior of the network traffic thereby aiding in network management. In this work, the traffic models considered are all of the ON-OFF type. However, the distributions vary. The cell arrival pattern is therefore bursty. All the traffic source models here have a 0.5 probability of being in the ON state. This also implies that the probability of being in the OFF state is 0.5 . Each of the sources generates traffic at the respective rates of 100 cells/s.

The models considered in this work include the Interrupted Poisson Process (IPP) in which the distribution of cells within each burst assumes a Poisson fashion (each arrival instant can only have an arrival and the instants are random); the Interrupted Bernoulli Process (IBP) in which distribution is Bernoulli fashioned (simultaneous arrivals can be accommodated within an arrival instant); the ON-OFF (constant distribution) model in which the distribution of cells within a burst assumes a constant trend. These models are shown in the figures below: 


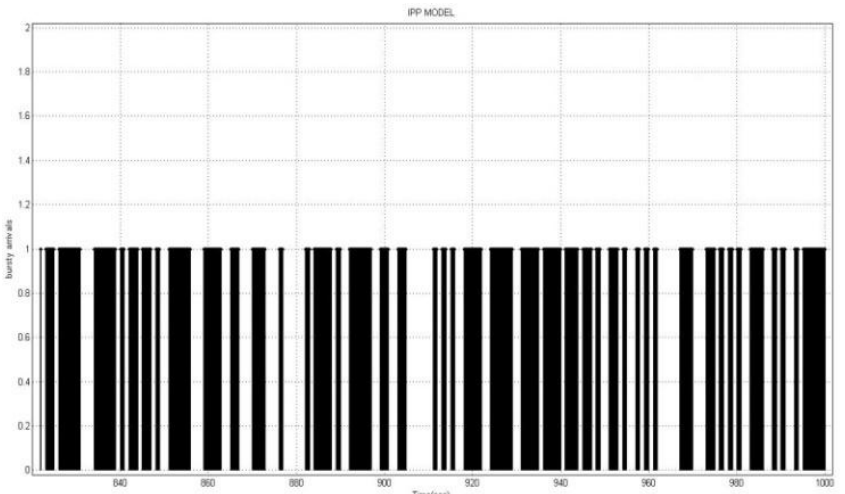

Fig 1: The Interrupted Poisson Process model.

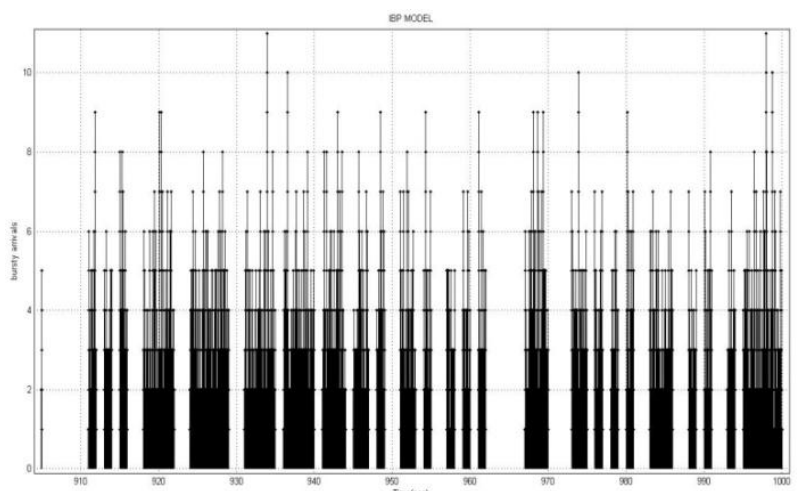

Fig 2: The Interrupted Bernoulli Process model.

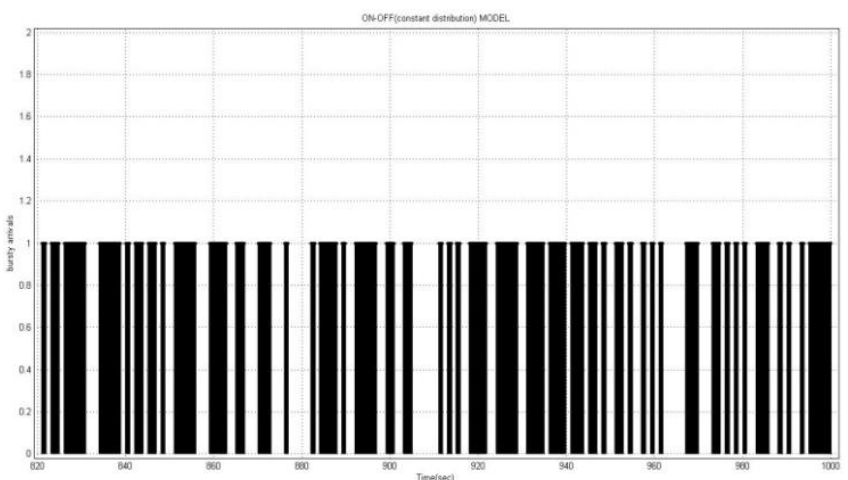

Fig 3: The ON-OFF (constant distribution) model.

In interrupted Poisson process (IPP), the ON period is referred to as the ACTIVE period. This is the period during which arrivals occur in a Poisson fashion. On the other hand, the OFF period is referred to the INACTIVE or IDLE period during which no arrivals occur. These two periods are exponentially distributed, and they alternate continuously. In interrupted Bernoulli process (IBP), arrivals occur during the ON period in a Bernoulli fashion while no arrivals occur during the OFF period. The two periods, are geometrically distributed. But for the ON-OFF (constant distribution) model, as the name implies, the distribution of the ON and OFF periods assumes a constant fashion.

\section{Simulation Results And Analysis}

Buffer occupancy and resource utilization: The buffer being one of the resources of the network is very important in analyzing what happens inside the network. If it is fully occupied, the implication is that new arrivals will be lost. As shown in the figure 4, the IBP model gives the highest level of buffer occupancy which also implies that it would record higher losses than any other one considered. The IPP traffic model has a very good buffer occupancy factor while the ON-OFF model with constant distribution gives the best result. Similar result is shown in figure 5 which gives the resource utilization. 


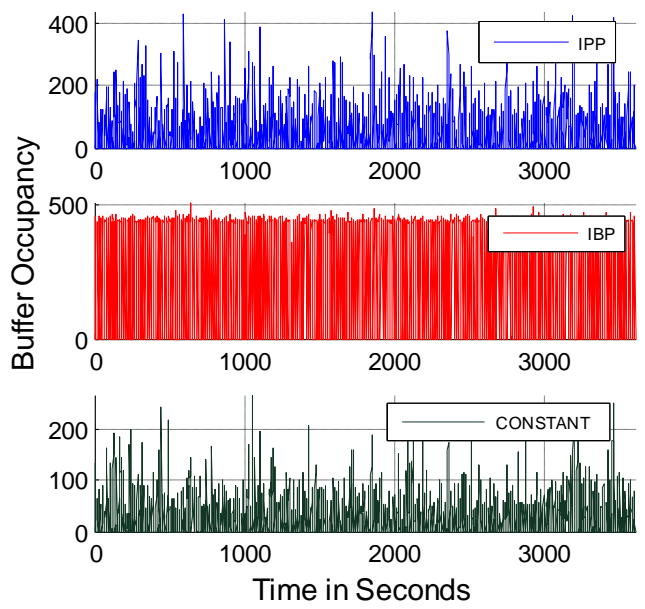

Fig 4: Buffer occupancy

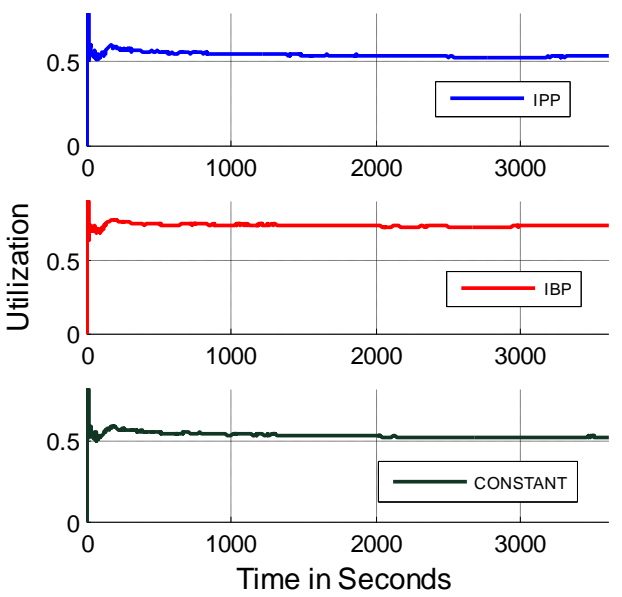

Fig 5: Utilization

Queuing Delay: Real time applications do not tolerate delay. So the traffic model to adopt should be the one with minimal delay factor. From figure 6, it is obvious that the IBP model experiences more delay than the any of the other two models considered. The overall implications of this being that for real time applications, using an IBP traffic model would defeat the intended objectives. The resources involved in such a process are wasted. Of the three models considered, the ON-OFF model with Constant distribution gives the smallest delay and as such, is most suited for real-time applications.

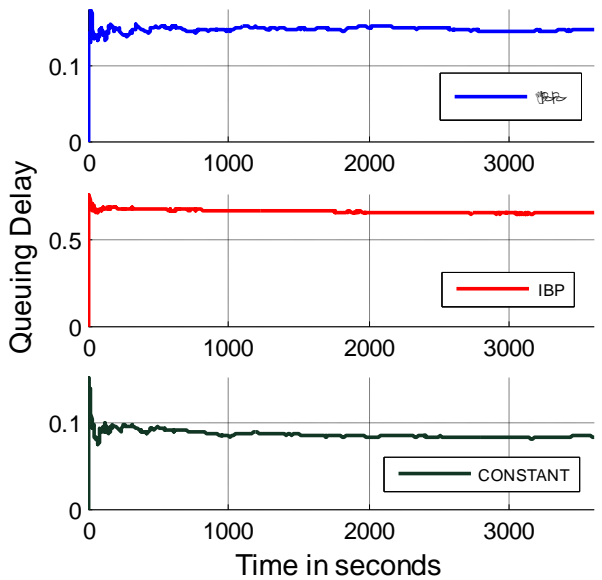

Fig 6: Cell delay 
Economic Implications: Using these three metrics, the traffic models considered can then be evaluated economically to ascertain the economic implications of using any of the models. To the network service provider, the target is to be able to provide the needed services with satisfactory quality and at the same time make profits. One of the major contributing factors of service dissatisfaction is congestion. When the network channel becomes congested, quality of services rendered becomes degraded and when quality degrades, demand falls and when demand falls, profit goes low. So this economic chain is of particular interest to the network designer. The traffic models considered in this work have different contributions to network congestions as suggested in the figures above. The order of contribution is IBP, IPP and ON-OFF (with Constant distribution). Consequently, bursty traffic models fashioned as IBP has the worst case scenario while the one fashioned as $\mathrm{ON}-\mathrm{OFF}$ (with constant distribution) has the best performance.

\section{Conclusion}

We have been able to confirm that different arrival patterns affect burstiness. This is in line with previous works done in the area. We have also shown that among the three models considered in this work, the ON-OFF (with constant distribution) is more economical in network design. In our subsequent study, we will investigate the effect of varying cell arrival patterns on the Dynamic call admission control mechanism. Our particular interest would be to consider the QoS metrics alongside the DCAC.

\section{References}

[1]. Cruz R. L., "Quality of Service Guarantees in Virtual Circuit Switched Networks", IEEE Journal of Selected Areas in Communication, 13(6): pp 1048-1056, 1995.

[2]. Ferrari D. \& Verma D., "A Scheme for Real-Time Channel Establishment in Wide-Area Networks", IEEE Journal of Selected Areas in Communication, vol. 8. Pp 368-379, April 1990

[3]. Wang Z. \& Crowcroft J., "Analysis of Burstiness and Jitter in Real-Time Communications", The Proceedings of SIGCOMM, PP 13-19, 1993

[4]. Park K., Willinger W. (editors), "Self-Similar Network Traffic and Performance Evaluation”, John wiley \& sons, 2000

[5]. Takine, T.; Okazaki, K. \& Masuyama, H., " IP Traffic Modeling: Most Relevant Time-Scale and Local Poisson Property", Department of Applied Mathematics and Physics Kyoto University. (ICKS'04) Informatics Research for Development of Knowledge Society Infrastructure, 2004

[6]. Paulo H. P. de Carvalho, Marcio A. de Deus and Priscila S. Barreto, "IP and 3G bandwidth management strategies applied to capacity planning", in Telecommunications Network- Current Status and Fututre Trends; pp 29-50

[7]. Ayyorgun S. and Feng W., "A Probablisitic Definition of Burstiness Characterization: A Systematic Approach", Research and Development in Advanced Network Technology (RADIANT), May 2003

[8]. Perros H.G, "Call Admission Control Schemes: A Review,” IEEE Communications Magazine, Nov 1996

[9]. Saito H, Kawarasaki M, and Yamada H, "An Analysis of Statistical Multiplexing in an ATM Transport Network," IEEE JSAC, Vol.9, No 3, April 1991

[10]. Saito H, "The Departure Process of an N/G/1 Queue," Performance Evaluation, Vol.111, pp 241-251, 1990

[11]. Kang S.H, Kim Y. H, Sung D. K, and Choi B. D, “An Application of Markovian Arrival Process (MAP) to Modeling Superposed ATM Cell Streams," IEEE Transactions on Communications, vol. 50, No4, April 2002 